\title{
Object and scene-centric activity detection using state occupancy duration modeling
}

\author{
Murtaza Taj and Andrea Cavallaro* \\ Queen Mary, University of London \\ Mile End Road, London E1 4NS (UK) \\ \{murtaza.taj, andrea.cavallaro\}@elec.qmul.ac.uk
}

Paper ID 66

\begin{abstract}
We propose a video event analysis framework based on object segmentation and tracking, combined with a Hidden Semi-Markov Model (HSMM) that uses state occupancy duration modeling. The observations generated by a multiobject detector and tracker are used as emitting symbols and the corresponding probabilities are computed using multivariate Gaussians. Next, we recognize events by estimating the most likely object state sequence using a HSMM decoding strategy, based on the Viterbi algorithm. Moreover, the duration distribution enforces the state transition after certain time and hence better models the events constrained on time intervals. We demonstrate and evaluate the proposed framework on a dataset of approximately $20 \mathrm{~K}$ frames, and show that the duration modeling improves the event detection results by $7 \%$ to $11 \%$, compared to state-ofthe-art HMMs.
\end{abstract}

\section{Introduction}

The automated analysis of large volumes of data is of great interest for indexing and retrieval of surveillance videos. Algorithms capable of detecting objects and events of interest based on motion patterns and semantic understanding are highly desirable to summarize videos or to trigger alarms.

Video event detection algorithms can be classified into three main groups, namely 3-D model-based, temporal templates and trajectory-based. 3D model-based approaches treat an object as a set of connected parts and perform detections on their activities ([4]). The activities can be

\footnotetext{
* This work was supported in part by the EU, under the FP7 project APIDIS (ICT-216023).
}

modeled as generalized action cylinders ([15]) or volumetric features ([13]). Temporal templates use sequences of simple events to model more complex events. Examples of temporal template methods are Petri Nets ([9]) and Belief Networks ([12]). Activity and plan prototypes are also used to recognize object behaviors through perceptual processing ([6]). A temporal template generated using recency of motion in a sequence can also be used for complex event recognition ([8]). Trajectory-based techniques perform event detection by analyzing trajectories over certain time spans ([10]). Abnormal behaviors can be detected by performing outliers detection using unsupervised clustering ([3]). Since events are generally composed of specific sequences of operations, HMMs are appropriate to model them ([22]). Hidden Markov Models are also used to perform abnormal activity detection in crowds ([2]) by modeling normal motion paths using single HMM ([2]) or Mixture of Gaussian HMMs ([1]). The main limitation of the above mentioned HMM-based techniques is the use of an evaluation strategy to obtain the sequences of events, as this result in a dependence on the selected pattern.

The event detection problem can be decomposed into three main steps: (i) the extraction of objects of interest, (ii) the tracking of the objects, and (iii) the detection of events generated by the tracked objects. As event detection can be modeled as a random process that is segmental in nature, the piecewise stationarity assumption of Hidden Markov Models (HMMs) is well suited for event modeling.

In this paper we improve the video event analysis approach of [20] by using Hidden Semi-Markov Model (HSMM) with time distribution modeling. The time distribution allows to incorporate in the model the dependency on time for triggering events and enables a smoother state transition than a thresholded decision. Moreover, the proposed approach can be applied as object-centric or scenecentric model to better fit the events of interest. The 
object-centric approach is used to model events associated to objects whereas the scene-centric approach suits well for environment-dependent events.

The paper is organized as follows. Section 2 provides an overview of our detection and tracking algorithms used to extract the objects of interest. The proposed event detection approach is described in Section 3, followed by the experimental results that are presented in Section 4. Finally, in Section 5 we draw the conclusions.

\section{Object extraction and tracking}

Let an object detection module generate a set of $R$ objects $O_{t}=\left\{O_{t}^{1}, O_{t}^{2}, \cdots, O_{t}^{R}\right\}$ at time $t$. The problem is to associate objects between consecutive frames to establish the track $X_{t}^{r}=\left\{O_{t_{0}}^{r} \ldots O_{t}^{r}\right\}$, up to time $t$, of each object $O_{t}^{r}$. The event analysis is then performed based on the tracks and on the available contextual information.

We perform video object extraction (foreground segmentation) using a statistical color change detector ([7]), a model-based algorithm that assumes additive white Gaussian noise introduced by the camera. The noise amplitude is estimated for each color channel separately. Given a reference image (i.e., an image without objects or an image generated by an adaptive background algorithm ([19])), the algorithm removes the effect of the camera noise based on the hypothesis that the additive noise affecting each image of the sequence respects a Gaussian distribution, with zero mean and standard deviation $\sigma_{t}$. The value of $\sigma_{t}$ is computed on-line by analyzing the image difference in areas without moving objects. After the background/foreground classification, any isolated noise pixel is removed using dilation and erosion.

An important problem is that moving vegetation and fast illumination changes reduce the accuracy of the object extraction results by introducing false positive detections. For this reason, we filter the detections using a Probability Hypothesis Density filter (PHD filter) ([14]), which helps eliminating temporally inconsistent false positives and smoothing the results of the detections (Figure 1). Once the objects are extracted, we associate objects across consecutive frames in order to establish the track $X_{t}^{r}$ for object $r$ up to time $t$. The trajectory $X_{t}^{r}$ is estimated with a graph matching algorithm ([21]).

Let $\left\{X^{r}\right\}_{r=1 \ldots R}$ be a set of $R$ object detections, $v\left(\mathbf{x}_{i}^{\alpha}\right) \in$ $V_{i}$ the set of vertices representing the detected objects at time $i$, and $e\left(v\left(\mathbf{x}_{i}^{\alpha}\right), v\left(\mathbf{x}_{j}^{\beta}\right)\right) \in E$ the set of edges of the graph $G(V, E)$. Edges represent all possible track hypotheses. Each $v\left(\mathbf{x}_{i}^{\alpha}\right)$ belongs to $D$, a bi-partitioned digraph (i.e., a directional graph). The candidate correspondences at different observation times are described by the gain $g$ associated to the edges that link the vertices. The best set of tracks is computed by finding the maximum weighted path cover of $G$. This step can be performed using the algorithm by

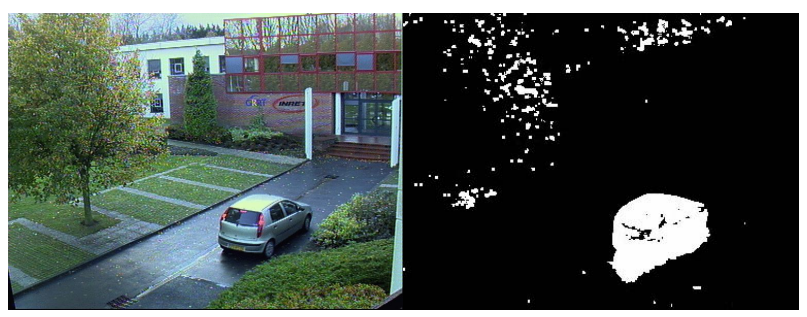

(a)

(b)

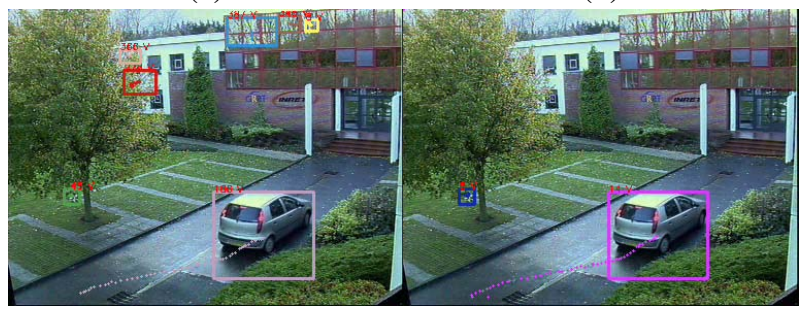

(c)

(d)

Figure 1. Comparison of object extraction results with and without the PHD Filter. (a) Original frame; (b) change detection result without using the PHD Filter; (c) bounding boxes of the detected objects without using the PHD Filter (6 false detections); (d) bounding boxes of the filtered objects after applying the PHD Filter ( 5 false detections have been removed).

Hopcroft and Karp ([11]) with complexity $O\left(n^{2.5}\right)$, where $n$ is the number of vertices in $G$. After the maximization procedure, a vertex without backward correspondence models a new object, and a vertex without forward correspondence models a disappeared object. The depth of the graph $K$ determines the maximum number of consecutive miss detected or occluded frames during which an object track can still be recovered.

The gain $g$ between two vertices is computed using the information in $X_{i}$, where the elements of the set $X_{i}$ are the vectors $\mathbf{x}_{i}^{\alpha}$ defining $\mathbf{x}$, the state of the object $\mathbf{x}=$ $(x, y, \dot{x}, \dot{y}, h, w, H)$, where $(x, y)$ is the center of mass of the object, $(\dot{x}, \dot{y})$ are the vertical and horizontal velocity components, $(h, w)$ are the height and width of the bounding box, and $H$ is the color histogram. The gain for each couple of nodes, $\left(\mathbf{x}_{i}^{\alpha}, \mathbf{x}_{j}^{\beta}\right)$, is computed based on the position, direction, appearance and size of a candidate object ([21]).

This process results in the track $X_{t}^{r}$ for each object $r$. The tracks of the objects are then used for event analysis, as described in the next section.

\section{Event analysis}

\subsection{HSMM with duration modeling}

Let $\lambda=\{A, B, \omega\}$ be a continuous distribution firstorder Hidden Markov Model, where $\omega=\left\{\omega_{1}, \cdots, \omega_{N}\right\}$ represents the events (states) to be detected (we denote the actual state at time $t$ as $\omega(t)) ; A=\left\{a_{i j}\right\}$ represents the state 

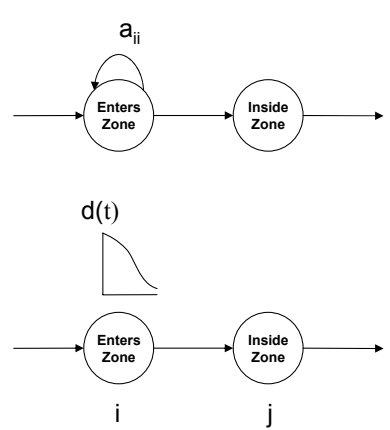

Figure 2. Examples of self-transition modeling for an Hidden Markov Model: (top) self-transition probability $\left(a_{i i}\right)$; (bottom) self-transition replaced with a state occupancy duration pdf.

transition probabilities, with $a_{i j}=P\left[\omega(t+1)=\omega_{j} \mid \omega(t)=\right.$ $\left.\omega_{i}\right], \quad 1 \leq i, j \leq N ; B=\left\{b_{j t}\right\}$ represents the emission probabilities, with $b_{j t}=P\left[O_{t}^{r} \mid \omega(t)=\omega_{j}\right]$. The emitting symbols of each state are provided by the track $X_{t}^{r}$ of the observation $O_{t}^{r}$ of object $r$.

Given the model $\lambda$ and the observation sequence, we can obtain the associated optimal state sequence. Given the probability of the best sequence up to time $t$, the single most probable state sequence $\omega(t+1)$ at time $t+1$ can be obtained as

$$
\delta_{i}(t)=\max _{\Omega_{t-1}} P\left(\Omega_{t-1}, \omega(t)=\omega_{i}, O_{1} \cdots O_{T}\right),
$$

where $\Omega_{t-1}=\{\omega(1) \cdots \omega(t-1)\}$ and $O_{1} \cdots O_{T}$ are the observations from time 1 to $T$.

$$
\delta_{i}(t)=\max _{1 \leq t \leq T-1} P\left(\omega(t)=\omega_{i} \mid O, \lambda\right) .
$$

According to the Markovian assumption, the conditional probability distribution of future states depends on the current state only and not on past states, hence using the Forward Viterbi we have

$$
\delta_{j}(t+1)=\max _{1 \leq i \leq N}\left[\delta_{i}(t) a_{i j}\right] b_{j, O^{l}} . \quad 1 \leq l \leq T
$$

Finally, we compute the most likely hidden state sequence $\omega_{T}$ up to time $t+1$ as

$$
\omega(t+1)=\arg \max _{1 \leq i \leq N}\left[\delta_{i}(t) a_{i j}\right] .
$$

This simple Hidden Markov Model is unable to completely model certain events due to the duration distribution of the observation sequence for a certain state. The Markovian assumption constraints the state occupancy distribution to be exponentially distributed ([16]). Therefore the estimation of the most likely path $\omega_{T}$ is problematic, because a state with high self-transition probability $a_{i i}$ can cause the algorithm to stay in this state for a longer interval. To avoid such self-transitions, we use Hidden Semi-Markov Models
(HSMM) ([18]) to enable the explicit modeling of duration probability distribution $d(t)$. The duration probability distribution is the probability of staying at least for a duration $\tau$ in the state $\omega_{j}$, with $1 \leq \tau \leq D_{j}$ (Figure 2). To compute the most likely state sequence $\omega_{T}$ using the durational distribution, we maximize the joint probability $P\left[O, \omega_{T}^{1} \mid \lambda\right]$ by re-writing Equation 2 as

$$
\delta_{i}(t)=\max _{O, 1 \leq t \leq T-1} P\left[\omega(t)=\omega_{i}, O \mid \lambda\right] .
$$

Using the forward Viterbi algorithm we can solve Equation 3 as

$$
\delta_{j}(t+1)=\max _{\substack{1 \leq j \leq N \\ 1 \leq \tau \leq D_{j}}}\left[\max _{1 \leq i \leq N}\left[\delta_{i}(t) a_{i j}\right] d(t) b_{j, O^{l}}\right]
$$

with $1 \leq l \leq T$. Given the model $\lambda=\{A, B, \omega\}$ and the duration probability distribution $d(t)$, we can now use Equation 6 to compute the best state sequence by performing the HMM decoding using the Viterbi algorithm. The state transition probabilities $a_{i j}$ can be defined empirically or, if there is sufficient training data, can be calculated using the Baum-Welch algorithm ([5]). In order to use the Viterbi algorithm we need first to model the duration probability distribution $d(t)$ and the observation sequence.

\subsection{Duration probability distribution}

The duration probability distribution $d(t)$ can be modeled using different parametric duration distributions. We evaluate two distributions, namely the half-normal distribution and the triangular distribution, which are well adapted to the problem at hand. The half-normal distribution, $d_{n}(t)$, can be expressed as

$$
d_{n}(t)=\frac{1}{\sigma} \sqrt{\frac{2}{\pi}} \exp \left(-\frac{1}{2}\left(\frac{t-\mu}{\sigma}\right)^{2}\right),
$$

where $\sigma$ is the variance, computed as $3 \sigma=\tau$, and $\mu$ is the mean. The mean is the time $t_{e}$ when the object transits into the state and $t_{e} \leq t \leq T+t_{e}$. The triangular distribution, $d_{t}(t)$, can be expressed as

$$
d_{t}(t)=\frac{2\left(\tau+t_{e}-t\right)}{\tau^{2}} .
$$

In case of events with high self-transitions a uniform distribution can be used which implicitly converts HSMM to HMM. The selection of the appropriate distribution, for the specific event or activity, can be done using Chi-square test. The evaluation and analysis of the results obtained using half-normal and triangular distributions are discussed at the end of Section 3.3 and in Section 4. 


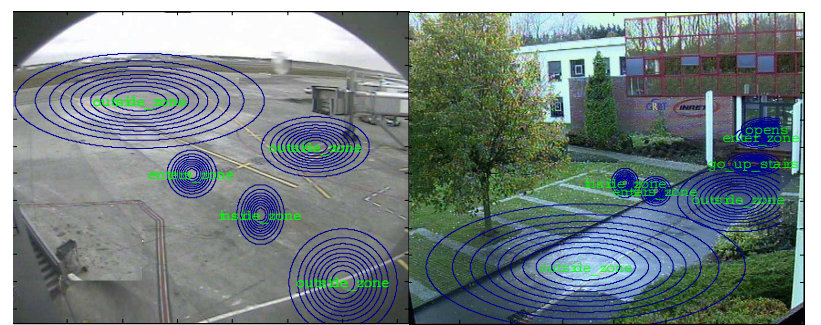

(a)

(b)

Figure 3. Multivariate scene-centric distribution model. (a) Sequence AP-11 C4; (b) Sequence BE-19 C1.

\subsection{Object-centric and scene-centric models}

For the observation sequence model, the emitting symbol is the observation of the $r^{t h}$ object $O_{t}^{r}$ at time $t$. The emission probabilities $b_{j t}$ are modeled as a continuous function describing the state. We propose and evaluate two models to estimate $b_{j t}$, namely a scene-centric and an object-centric model. In the scene-centric approach, the $b_{j t}$ are modeled as a multivariate Gaussian. For each $j^{\text {th }}$ state we use a multivariate Gaussian $\mathcal{N}_{j}(\mu, \Sigma)$ with mean $\mu$ and covariance $\Sigma$ as

$$
b_{j t}=\frac{1}{\sqrt{(2 \pi)^{n}\left|\Sigma_{j}\right|}} \exp \left(-\frac{1}{2}\left(X-\mu_{j}\right)^{T} \Sigma_{j}^{-1}\left(X-\mu_{j}\right)\right),
$$

where $n=4, \mu_{j}=\left\{\mu_{x}, \mu_{y}, \mu_{w}, \mu_{h}\right\}$ and $\left|\Sigma_{j}\right|$ is the determinant of the covariance matrix $\Sigma_{j}$, which we assume to be a diagonal matrix: $\Sigma_{j}=\operatorname{diag}\left[\sigma_{x}^{2}, \sigma_{y}^{2}, \sigma_{w}^{2}, \sigma_{h}^{2}\right]$. The values for $\mu_{j}$ and $\Sigma_{j}$ are set based on the contextual information specific to the task at hand (Figure 3).

In the object-centric approach, we model $b_{j t}$ as a multivariate distribution composed of a mixture of a normal and a uniform distribution $\mathcal{N}_{j}(\mu, \Sigma, \rho, C, D)$ with mean $\mu$, covariance $\Sigma$, weight $\rho$ and range of uniform distribution $[C, D]$ :

$$
\begin{array}{r}
b_{j t}=\frac{\rho}{(2 \pi)^{\frac{K}{2}}\left|\Sigma_{j}\right|^{\frac{1}{2}}} \exp \left(\sum_{k=1}^{K}\left[\frac{\left(\theta_{k}-\mu_{\theta_{k}}\right)^{2}}{2 \sigma_{\theta_{k}}^{2}}\right]\right)+ \\
+\frac{(1-\rho)}{\pi} \prod_{k=1}^{K}\left[\frac{\psi_{\theta_{k}}}{\sigma_{\theta_{k}}}\right],
\end{array}
$$

where $K=2 ; \theta_{1}=x$ and $\theta_{2}=y$. Therefore $\sigma_{x}$ and $\sigma_{y}$ are the standard deviations, respectively. The functions $\psi_{k}$ are piecewise binary and defined as

$$
\psi_{x}=\left\{\begin{array}{ll}
1 & \text { if } C_{x}<x<D_{y} \\
0 & \text { otherwise }
\end{array},\right.
$$

and

$$
\psi_{y}= \begin{cases}1 & \text { if } \zeta\left(C_{x}\right)<y<\zeta\left(D_{y}\right) \\ 0 & \text { otherwise }\end{cases}
$$

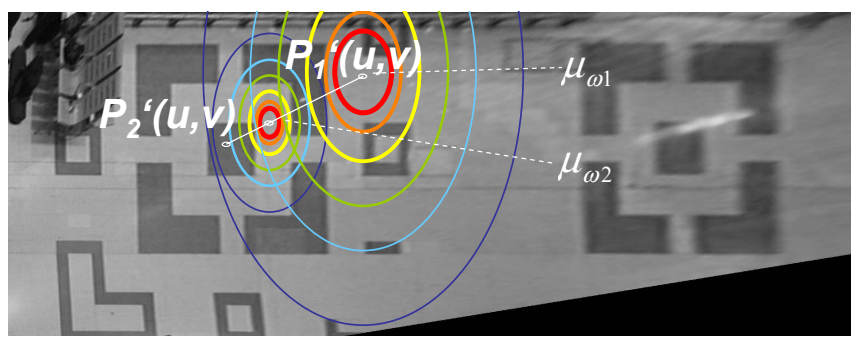

Figure 4. Multivariate object-centric distribution model. The distribution of the states is placed on the line joining the centroids of the objects.

where $\zeta= \pm \sigma_{y} \sqrt{1-\left(\frac{x-x_{c}}{\sigma_{x}}\right)^{2}}+y_{c}$, with $\left(x_{c}, y_{c}\right)$ representing the object centroid around which the model is built, and $\pi \prod_{k=1}^{2} \sigma_{\theta_{k}}$ is the area of an ellipse. $\left|\Sigma_{j}\right|$ is the determinant of the covariance matrix, with $\Sigma_{j}=\operatorname{diag}\left[\sigma_{x}^{2}, \sigma_{y}^{2}\right]$ and therefore $\left|\Sigma_{j}\right|=\sigma_{x} \sigma_{y}$ in Equation 10. The values of the elements in $\Sigma_{j}$ depend on the state to be modeled, whereas the value of $\mu$ is assigned dynamically. This is the key point of the proposed object-centric modeling. The value of $\mu$ of the first state is set as the centroid of the reference object $O_{t}^{r e f}$ (Figure 4). The remaining state distributions are then placed around $O_{t}^{r e f}$ to estimate the possible state of $O_{t}^{r e f}$ with respect to the objects $O_{t}^{r}$. The $\mu$ of the other states are positioned on the line passing through the centroid of the two objects $\left(O_{t}^{r e f}\right.$ and $\left.O_{t}^{r}\right)$ at a distance that is a function of the variances of the states to be detected. The rationale for using Gaussian functions instead of hard boundaries and fixed threshold is to increase the flexibility of the algorithm in order to detect several different events in different scenarios.

Let us see two examples of event modeling using the scene-centric and the object-centric models for the PETS ${ }^{1}$, the CAVIAR ${ }^{2}$ and the ETISEO ${ }^{3}$ datasets. We use the objectcentric HMM shown in Figure 5 to model the events in the PETS and the CAVIAR datasets. In this case, we model three events, namely the attended, unattended and abandoned baggage event. For the PETS sequences, the baggages are detected based on their size and aspect ratio (ranging between 1 and 1.8). For the attended baggage $\left(\omega_{1}\right)$ event, $\sigma_{x}=\sqrt{2 * 36}$ and $\sigma_{y}=\sqrt{2 * 96}$ respectively, whereas for the unattended baggage $\left(\omega_{2}\right)$ and the abandoned baggage $\left(\omega_{3}\right)$ events the values are $\sigma_{x}=\sqrt{36 / 2}$ and $\sigma_{y}=\sqrt{96 / 2}$. These values are based on the calculation that, for this scenario, $1 \mathrm{~m}$ in world-coordinates corresponds in the ground plane to 36 pixels along the $x$-axis and to 96 pixels along the $y$-axis. A baggage is considered unattended when its related object (the owner) is $2 m$ away. A baggage is considered abandoned when its related ob-

\footnotetext{
${ }^{1}$ http://www.cvg.rdg.ac.uk/PETS2006/index.html

${ }^{2}$ http://homepages.inf.ed.ac.uk/rbf/CAVIARDATA1/

${ }^{3}$ http://www.silogic.fr/etiseo/index.html
} 


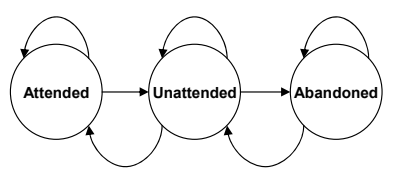

Figure 5. HMM model for baggage detection on the PETS and CAVIAR datasets. Each state represents an event. The initial state is selected as the state with the maximum emission probability $b_{j t}$ at time $t_{0}$.

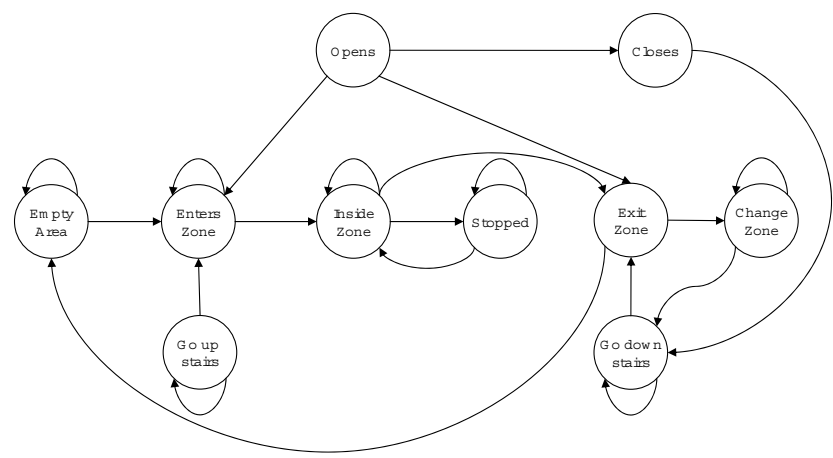

Figure 6. Scene-centric HMM model for activity monitoring on the ETISEO dataset. Each state represents an event. The initial state is selected as the state with the maximum emission probability $b_{j t}$ at time $t_{0}$.

\begin{tabular}{|r|r|r|r|}
\hline & HMM & HSMM-TRI & HSMM-HN \\
\hline AP & 0.882 & 0.980 & 0.956 \\
\hline BE & 0.790 & 0.966 & 0.980 \\
\hline Total & 0.847 & 0.965 & 0.975 \\
\hline
\end{tabular}

Table 1. Performance comparison between the proposed HSMM algorithm with half-normal and triangular distribution for state occupancy duration and event detection using HMM without state duration modeling.

ject is $3 m$ away, for at least 30 seconds. For the CAVIAR sequences, the baggages are detected in a similar fashion and the parameters of the events are defined as follows. For the attended baggage $\left(\omega_{1}\right)$ event, $\sigma_{x}=\sqrt{2 * 36}$ and $\sigma_{y}=\sqrt{2 * 36}$ respectively, whereas for unattended baggage $\left(\omega_{2}\right)$ the values are $\sigma_{x}=\sqrt{36}$ and $\sigma_{y}=\sqrt{48}$ and for abandoned baggage $\left(\omega_{3}\right)$ the values are $\sigma_{x}=\sqrt{24}$ and $\sigma_{y}=\sqrt{24}$.

The scene-centric HMM model is used for activity monitoring for the ETISEO dataset (Figure 6). In this case we model ten events, namely enter zone, inside zone, exit zone, change zone, opens, closes, go up stairs, go down stairs, empty area, and stopped object. The definition of the areas of interest is part of the contextual information provided with the dataset. The most likely state sequence $\omega_{T}^{r}$ for each object $r$ is computed by applying the forward Viterbi algorithm after every 25 to 50 observations. The last state $\omega(t)$ of the state sequence is then used as the initial state $\omega(0)$ for next computation. The event detection algorithm using

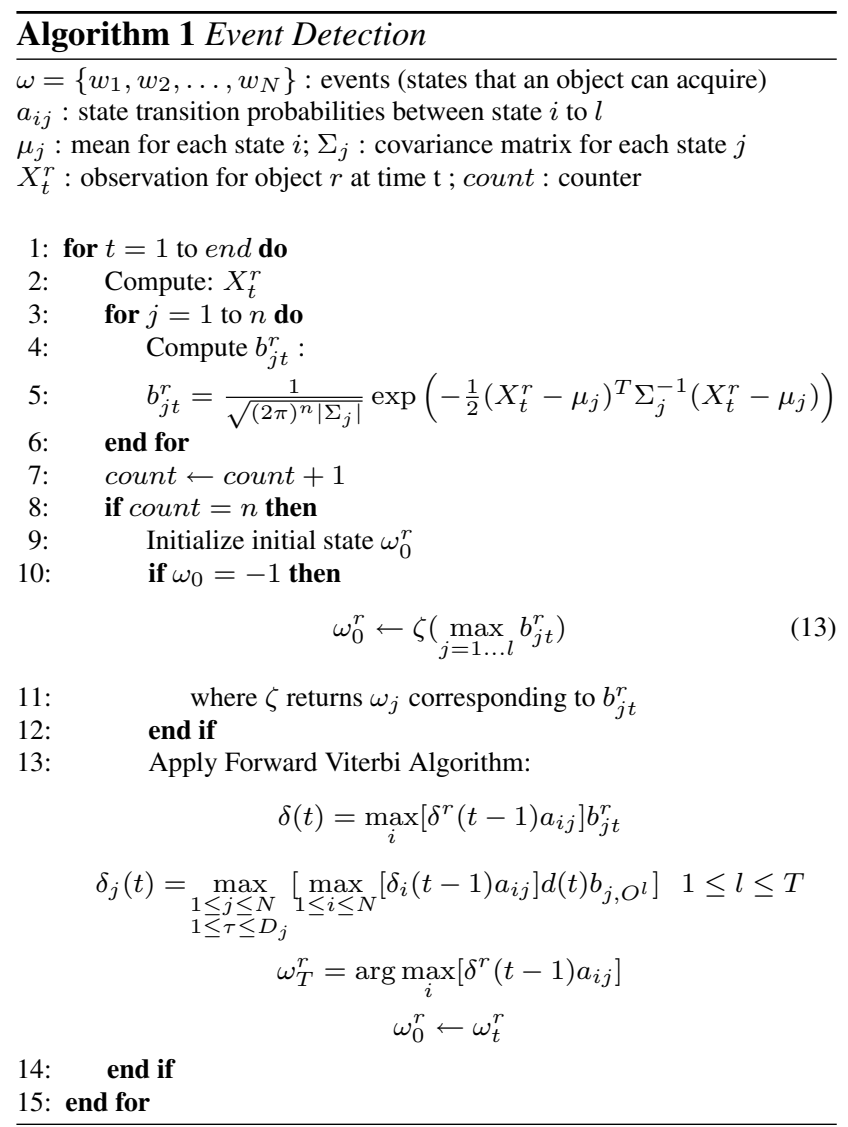

the forward Viterbi algorithm for HSMM is summarized in Algorithm 1.

Table 1 shows the performance comparison between the proposed algorithm (with the two different duration distributions) and the HMM-based algorithm without state duration modeling ([20]). The comparison was done on the ETISEO sequences AP-11 (C1 and C4) and BE-19 (C1) using the CREDS protocol which provides a weighted sum of the true positive, false positive and false negative detections ([17]). It is possible to notice that the duration modeling in HMM improves the results and that the modeling using the triangular distribution outperformed by $3.75 \%$ the half-normal distribution. In summary, the HSMM model with triangular distribution performed at 96\%, the HSMM model with half-normal distribution obtained a score of 92.5\% and the HMM scored $85 \%$.

\section{Experimental results}

We demonstrate the proposed algorithm on standard event detection sequences from the PETS 2006, CAVIAR ('leaving bags behind') and ETISEO datasets. These sequences (whose details are given in Table 2) include indoor and outdoor scenarios with pedestrians, vehicles, objects and their interactions. The PETS dataset contains 


\begin{tabular}{|c|r|r|r|r|r|}
\hline Dataset & Seq. & Cameras & Resol. & $\begin{array}{r}\text { N. of } \\
\text { frames }\end{array}$ & $\begin{array}{r}\text { Fr. } \\
\text { rate }\end{array}$ \\
\hline \hline ETISEO & AP-11 & C4, C7 & $720 \times 576$ & 805,805 & 12.5 \\
& BE-19 & C1, C4 & $768 \times 576$ & 1025,950 & 25 \\
& RD-6 & C7 & $720 \times 576$ & 1201 & 25 \\
\hline PETS & S1 & C3 & $720 \times 576$ & 3022 & 25 \\
& S3 & C3 & $768 \times 576$ & 2372 & 25 \\
& S5 & C3 & $720 \times 576$ & 3402 & 25 \\
& S6 & C3 & $720 \times 576$ & 2802 & 25 \\
\hline CAVIAR & CL1 & NA & $384 \times 288$ & 1441 & 25 \\
& CL2 & NA & $384 \times 288$ & 1357 & 25 \\
\hline \multicolumn{2}{|l|}{ Total number of frames } & 19182 & - \\
\hline
\end{tabular}

Table 2. Summary of the datasets used in the experiments.

high-quality sequences (duration: 94 to 136 seconds), the CAVIAR dataset contains low-resolution sequences of medium quality (duration: 54 to 57 seconds), and the ETISEO dataset contains sequences of lower quality (duration: 40 to 64 seconds).
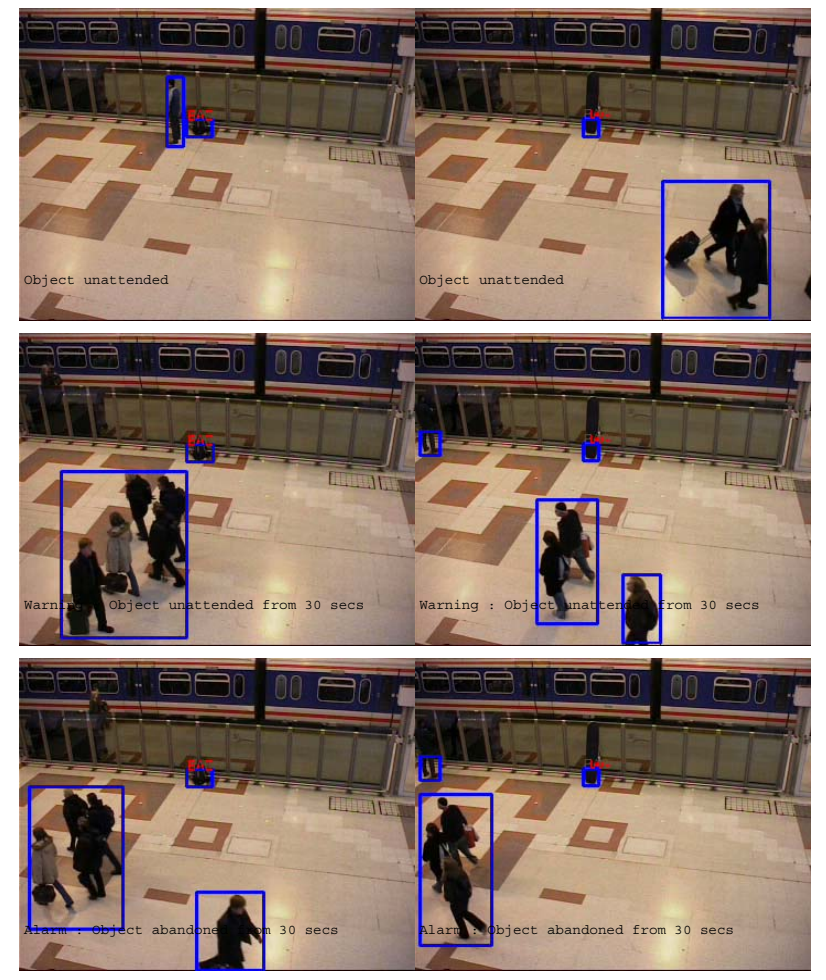

(a)

(b)

Figure 7. Sample event detection results from the PETS 2006 dataset using the object-centric event modeling. (a) Sequence S1 (frames 2004, 2754 and 2790); (b) Sequence S5 (frames 2083, 2833 and 2890).

To evaluate the performance of the event analysis results, we compute three measures: the accuracy, the precision and the sensitivity. Let $F P$ be the number of false positive detections, $T P$ the number of true positive detections, and $F N$ the number of false negative detections. More-

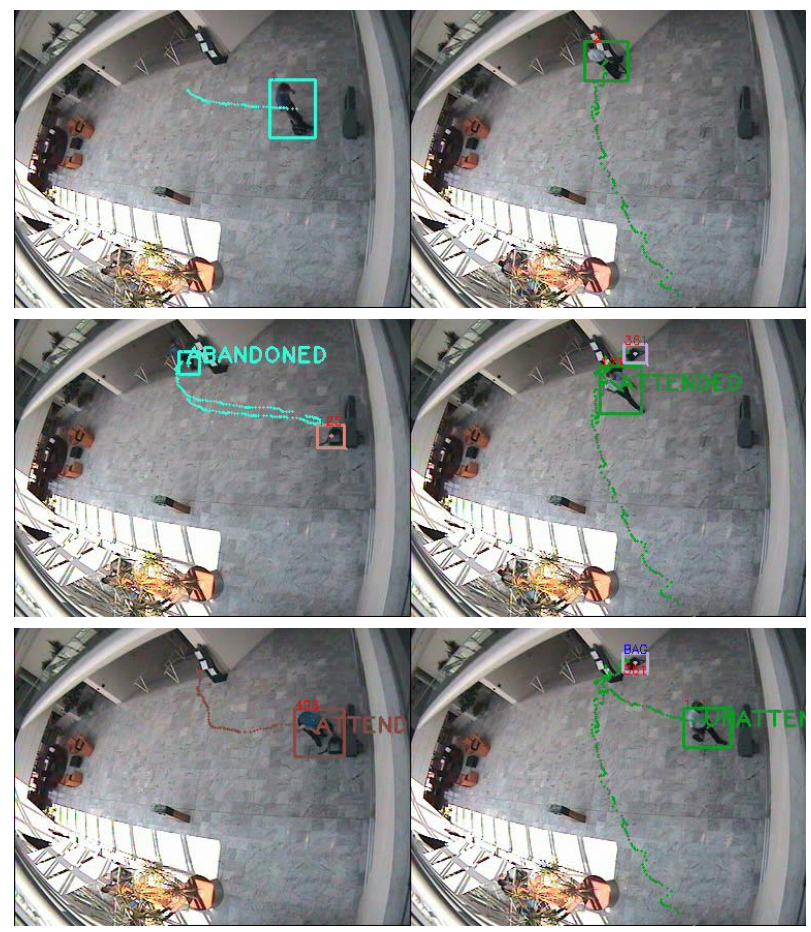

(a)

(b)

Figure 8. Example of left baggage detection on the CAVIAR dataset using the object-centric event modeling. (a) Abandoned and attended baggage event in sequence CL1 (frame 1014, 1070 and 1334); (b) attended and unattended baggage event in sequence CL2 (frame 548, 670 and 721).

over, let $G T$ be the starting or ending frame number corresponding to an event in the ground truth and $A D$ the corresponding frame number identified by the event detector for the same event. The accuracy quantifies the frame-level performance of the algorithm. The accuracy is defined as $\gamma=\left[1-\frac{|G T-A D|}{N F}\right] \times 100$, where $N F$ is a normalizing factor representing maximum allowed difference between AD and GT. Precision and sensitivity are sequence-level measures. The precision is the measure of the robustness against false positives. The sensitivity is the measure of the robustness against false negatives. The precision is defined as $T P /(T P+F P)$ and the sensitivity is defined as $T P /(T P+F N)$.

Figure 7 shows sample event detection results on the sequences S1 and S5 of the PETS 2006 dataset. The images show the detection of the object around which the model is built (the bag) and the subsequent sequence of events, namely a warning (unattended baggage) and an alarm (abandoned baggage). To evaluate the results, we computed the accuracy of the detection: for S1, the accuracy for the warning event is $90.5 \%$ and for the alarm event is $92.9 \%$; for $\mathrm{S} 3$, the accuracy is $100 \%$ for both events; 

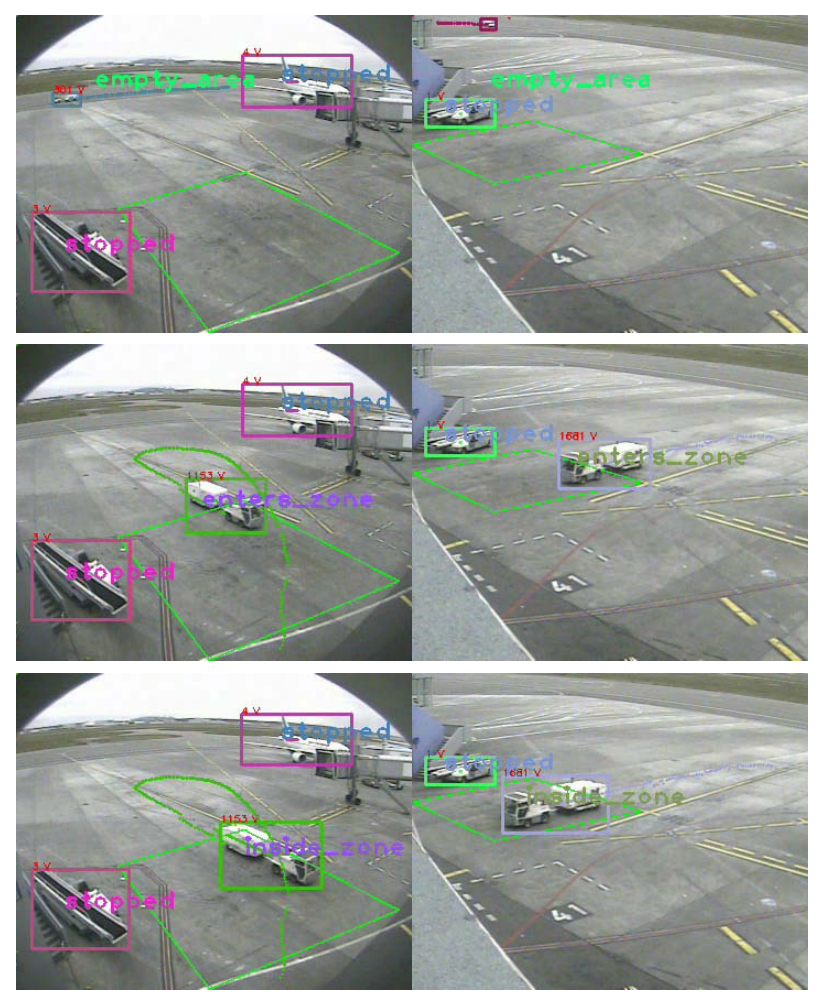

(a)

(b)

Figure 9. Sample tracking and event detection results for the ETISEO dataset using the scene-centric event modeling for ETIVS2-AP-11 (frame 23, 690 and 750). The detected events are stopped, empty area, enter zone and inside zone. (a) Camera 4; (b) Camera 7.

for S5, the accuracy is $88.8 \%$ and $83.02 \%$ for warning and alarm, respectively, and for S6 the accuracy is $98.5 \%$ and $95.5 \%$. Both precision and sensitivity for PETS are unitary as the object-centric approach selects events associated with detected objects only and the baggage was detected. Figure 8 shows sample event detection results on the sequences $C L 1$ and $C L 2$ of the CAVIAR dataset. Figure 8(a) shows the detection of the abandoned and attended baggage events, which are generated as the person first abandoned the baggage and then reappears and approaches the baggage. Figure 8(b) shows that the person has left the baggage at the end of the stairs moving toward the kiosk machine and hence the attended and then unattended baggage events are generated. In accordance with the ground truth available for the CAVIAR dataset, we compute the accuracy of the detection of the activities related to the baggage. For $C L 1$, the event initialization accuracy is $95 \%$ and the event termination accuracy is $94.66 \%$. The event initialization accuracy for CL2 is $97.33 \%$ and the termination accuracy is $95.60 \%$. The reason for these values is that the automated detection spans an interval that is a subset of the ground truth interval. This is due to the merging of the blob of the baggage with

\begin{tabular}{|c|c|c|c|c|c|c|}
\hline & \multicolumn{3}{|c|}{ Start frame } & \multicolumn{3}{|c|}{ End frame } \\
\hline & GT & AD & Acc & GT & AD & Acc \\
\hline \multicolumn{7}{|c|}{ AP-11-C4 (Precision: 1.00, Sensitivity: 0.56) } \\
\hline empty area & 1 & 12 & 98.53 & 689 & 664 & 96.67 \\
\hline enters zone & 675 & 664 & 98.53 & 720 & 728 & 98.93 \\
\hline inside zone & 690 & 731 & 94.53 & 804 & 803 & 99.87 \\
\hline stopped & 1 & 2 & 99.87 & 804 & 803 & 99.87 \\
\hline stopped & 1 & 3 & 99.73 & 804 & 803 & 99.87 \\
\hline All & & & 98.24 & & & 99.04 \\
\hline \multicolumn{7}{|c|}{ AP-11-C7 (Precision: 1.00, Sensitivity: 0.50 ) } \\
\hline empty area & 1 & 187 & 75.20 & 689 & 653 & 95.20 \\
\hline enters zone & 675 & 658 & 97.73 & 720 & 695 & 96.67 \\
\hline inside zone & 690 & 696 & 99.20 & 804 & 803 & 99.87 \\
\hline stopped & 1 & 2 & 99.87 & 804 & 803 & 99.87 \\
\hline All & & & 93.00 & & & 97.90 \\
\hline \multicolumn{7}{|c|}{ BE-19-C1 (Precision: 0.65, Sensitivity: 0.65) } \\
\hline closes & 335 & 371 & 95.20 & 453 & 450 & 99.60 \\
\hline opens & 258 & 250 & 98.93 & 320 & 300 & 97.33 \\
\hline opens & 366 & 395 & 96.13 & 400 & 407 & 99.07 \\
\hline stopped & 270 & 283 & 98.27 & 1025 & 1024 & 99.87 \\
\hline All & & & 97.13 & & & 98.97 \\
\hline \multicolumn{7}{|c|}{ BE-19-C4 (Precision: 0.87, Sensitivity: 0.35) } \\
\hline inside zone & 185 & 180 & 99.33 & 245 & 338 & 87.60 \\
\hline opens & 77 & 101 & 96.80 & 150 & 180 & 96.00 \\
\hline opens & 737 & 717 & 97.33 & 780 & 776 & 99.47 \\
\hline stopped & 170 & 206 & 95.20 & 950 & 1048 & 86.93 \\
\hline All & & & 97.17 & & & $\mathbf{9 2 . 5 0}$ \\
\hline \multicolumn{7}{|c|}{ RD-06-C7 (Precision: 1.00, Sensitivity: 0.25) } \\
\hline stopped & 570 & 559 & 98.53 & 710 & 743 & 95.60 \\
\hline All & & & 98.53 & & & 95.60 \\
\hline
\end{tabular}

Table 3. Event detection precision and sensitivity for 5 test sequences of the ETISEO dataset.

that of the person when the bag is placed on the floor. This results in a delayed detection of the event. Similarly, when the baggage is picked up, the two objects are merged thus resulting in an anticipation of the event. Improvements in the object detection accuracy will help in further enhancing the event detection accuracy. Similarly to the PETS dataset, the precision and sensitivity scores for the CAVIAR dataset are unitary as all events are detected.

Figure 9 shows detection results on the ETISEO dataset for the enter zone, inside zone, stopped and empty area events. The green rectangle drawn on the tarmac is the zone considered for triggering the events enter zone, inside zone and empty area. The stopped event is detected anywhere in the scene. Table 3 shows the accuracy for the detected events in all ETISEO sequences. The videos with the results for object tracking and event detection are available at http://www.elec.qmul.ac.uk/staffinfo/andrea/event.html. 


\section{Conclusions}

We presented an event detection framework based on object-centric and scene-centric Hidden Semi-Markov modeling (HSMM). First, multiple object extraction is achieved using color based change detection followed by a PHD filter and then graph theory is used for data association. Event detection is performed on the HSMM using the Viterbi decoding strategy to estimate the sequence of events performed by each object. We showed that HSMM has better capabilities of representing events than HMM due to the embedding of the state occupancy duration modeling, which not only resulted in an improvement in accuracy of $7 \%$ to $11 \%$, but also provides capabilities of detecting time constrained events without hard thresholds. We showed that the scene-centric approach can better model the activities associated to the contextual information, whereas activities related to the objects, irrespective of the environment, can be better modeled using the object-centric approach. The framework was evaluated on standard event detection datasets; with 19182 frames of indoor and outdoor standard test sequences.

Our current work addresses the use of proposed approach in multi-camera networks. Moreover, we will investigate the problem of event detection of multiple interacting objects.

\section{References}

[1] E. L. Andrade, S. Blunsden, and R. B. Fisher. Detection of emergency events in crowded scenes. In IEE Int. Symp. on Imaging for Crime Detection and Prevention, London, UK, June 2006.

[2] E. L. Andrade, S. Blunsden, and R. B. Fisher. Modelling crowd scenes for event detection. In Proc. of IEEE Conf. on Pattern Recognition, Hong Kong, China, August 2006. IEEE Computer Society.

[3] N. Anjum and A. Cavallaro. Unsupervised fuzzy clustering for trajectory analysis. In Proc. of IEEE Int. Conf. on Image Processing, San Antonio, Texas (USA), September 2007.

[4] C. Bregler, A. Hertzmann, and H. Biermann. Recovering non-rigid 3d shapes from image streams. In Proc. of IEEE Int. Conf. on Computer Vision and Pattern Recognition, South Carolina, USA, June 2000.

[5] D. Burshtein. Robust parametric modeling of durations in hidden markov models. IEEE Trans. on Speech and Audio Processing, 4(3):240-242, 1996.

[6] C. Castel, L. Chaudron, and C. Tessier. What is going on? a high level interpretation of sequences of images. In Proc. of the European Conf. on Computer Vision, Cambridge, UK, April 1996.

[7] A. Cavallaro and T. Ebrahimi. Interaction between high-level and low-level image analysis for semantic video object extraction. EURASIP Journal on Applied Signal Processing, 6:786-797, June 2004.

[8] J. W. Davis and A. F. Bobick. The representation and recognition of human movement using temporal templates. In Proc. of IEEE Int. Conf. on Computer Vision and Pattern Recognition, Washington, DC, USA, June 1997.

[9] N. Ghanem, D. DeMenthon, D. Doermann, and L. Davis. Representation and recognition of events in surveillance video using Petri nets. In Proc. of IEEE Int. Conf. on Computer Vision and Pattern Recognition, pages 112-112, Washington DC, USA, June 2004.
[10] S. Hongeng and R. Nevatia. Multi-agent event recognition. In Proc. of IEEE Int. Conf. on Computer Vision, pages 84-91, Vancouver, Canada, July 2001.

[11] J. Hopcroft and R. Karp. An $n^{2.5}$ algorithm for maximum matchings in bipartite graphs. SIAM J. Computing, 2(4):225-230, December 1973.

[12] S. S. Intille and A. F. Bobick. A framework for recognizing multiagent action from visual evidence. In Proc. of the National Conf. on Artificial intelligence, pages 518-525, Orlando, Florida, USA, September 1999.

[13] Y. Ke, R. Sukthankar, and M. Hebert. Efficient visual event detection using volumetric features. In Proc. of IEEE Int. Conf. on Computer Vision, volume 1, Beijing, China, October 2005.

[14] E. Maggio, E. Piccardo, C. Regazzoni, and A. Cavallaro. Particle phd filter for multi-target visual tracking. In ICASSP, pages I-1101 - I-1104, Honolulu, USA, April 2007.

[15] T. S. Mahmood, A. Vasilescu, and S. Sethi. Recognizing action events from multiple view points. In Proc. of IEEE Workshop on Detection and Recognition of Events in Video, Madison, Wisconsin, USA, June 2001.

[16] E. Marhasev, M. Hadad, and G. A. Kaminka. Non-stationary hidden semi markov models in activity recognition. In Proc. of the AAAI Workshop on Modeling Others from Observations, 2006.

[17] RATP, France. Call for Real-Time Event Detection Solutions (CREDS) for Enhanced Security and Safety in Public Transportation, July 2005.

[18] M. Russell and R. Moore. Explicit modelling of state occupancy in hidden markov models for automatic speech recognition. In Proc. of IEEE Int. Conf. on Acoustics, Speech, and Signal Processing, volume 10, pages 5-8, 1985.

[19] C. Stauffer and W. Grimson. Learning patterns of activity using realtime tracking. IEEE Trans. on Pattern Analysis and Machine Intelligence, 22:747-757, August 2000.

[20] M. Taj and A. Cavallaro. Multi-camera scene analysis using an object-centric continuous distribution hidden markov model. In Proc. of IEEE Int. Conf. on Image Processing, San Antonio, Texas (USA), September 2007.

[21] M. Taj, E. Maggio, and A. Cavallaro. Multi-feature graph-based object tracking. In CLEAR, Springer LNCS 4122, pages 190-199, Southampton, UK, April 2006.

[22] D. Zotkin, R. Duraiswami, and L. Davis. Multimodal 3-d tracking and event detection via the particle filter. In Proc. of IEEE Workshop on Detection and Recognition of Events in Video, pages 20-27, Vancouver, Canada, July 2001. 